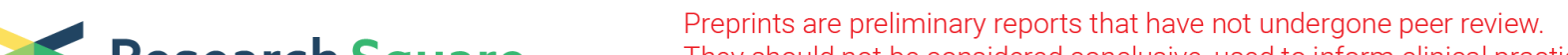 Research Square They should not be considered conclusive, used to inform clinical practice, or referenced by the media as validated information.
}

\section{M1A Regulator-Mediated Methylation Modification And Gene Signatures And Their Prognostic Value In Multiple Myeloma}

Jiaqi Fu

Shandong University of Traditional Chinese Medicine

\section{Xingjun Han}

The Second Affiliated Hospital of Shandong University of Traditional Chinese Medicine

\section{Wei Gao}

Shandong University of Traditional Chinese Medicine

Jinwei Cui

Jingwu Road Primary School of Jinan

Manya Yu

, Shandong University of Traditional Chinese Medicine

Xing Cui ( $\sim$ cdz45@foxmail.com )

The Second Affiliated Hospital of Shandong University of Traditional Chinese Medicine

\section{Research Article}

Keywords: m1A, multiple myeloma, methylation modification

Posted Date: February 16th, 2022

DOI: https://doi.org/10.21203/rs.3.rs-1338060/v1

License: (1) This work is licensed under a Creative Commons Attribution 4.0 International License.

Read Full License 


\section{Abstract}

Introduction: N1-methyladenosine (m1A), a methylation modification on RNA, is gaining attention for its role across diverse biological processes. However, the potential roles of m1A regulator-mediated methylation modification in multiple myeloma (MM) remain unclear.

Methods: We investigated the mRNA expression of m1A regulators in normal plasma $(N P, n=9)$ and MM $(n=174)$ bone marrow plasma cells and comprehensively evaluated the m1A modification patterns of $559 \mathrm{MM}$ samples based on the expression of $10 \mathrm{~m} 1 \mathrm{~A}$-related regulatory genes. A univariate Cox regression model, Kaplan-Meier survival curve, unsupervised clustering and gene enrichment analysis were used to explore the associations between m1A-related regulatory genes and MM patient survival and prognosis. Additionally, the m1Ascore was constructed to quantify m1A modification patterns of individual tumors using the principal component analysis algorithm.

Results: Compared with NP, the expression of five genes (TRMT61A, TRMT61B, YTHDF1, YTHDF2, YTHDF3) was upregulated in MM patients. The Kaplan-Meier survival curve showed the high expression of the above five genes was associated with shorter overall survival. Three distinct m1 A modification patterns were determined, of which cluster $B$ showed the worst outcome; the expression of ALKBH3, YTHDF2, TRMT10C, TRMT6, and TRMT61B was high and the expression of ALKBH1 and YTHDC1 was low in cluster $B$. In addition, the relationships between the m1 Ascore and survival, m1Aclusters, m1A gene clusters, and PD-L1 expression all indicated that high m1Ascore was associated with clinical benefits and therapeutic advantages.

Conclusion: These findings indicate that $\mathrm{m} 1 \mathrm{~A}$-related regulatory genes play a crucial role in regulating $\mathrm{MM}$ progression and that the m1Ascore could be used for diagnostic and prognostic purposes.

\section{Introduction}

Multiple myeloma (MM) is one of the most common malignant tumors in the blood system, with symptoms related to anemia, immunosuppression, bone destruction, and renal failure ${ }^{1}$. Myeloma biomarkers relevant to treatment can be both prognostic and predictive, and approaches to target these biomarkers are likely to have the greatest impact on outcomes ${ }^{3}$, so it is critical to identify novel biomarkers for early $\mathrm{MM}$ detection and prognostication.

The dynamic and reversible chemical modification of RNA plays a vital role in posttranscriptional gene regulation ${ }^{4}$. Decades of research have revealed more than 100 different types of posttranscriptional modifications to RNA molecules, including 5-methylcytosine (m5C), N6methyladenosine (m6A), and N1-methyladenosine (m1A). In the case of chemical conversion, m1A can undergo Dimroth rearrangement under alkaline conditions to become $\mathrm{m}^{6} \mathrm{~A}^{6}$. In contrast to $\mathrm{m} 6 \mathrm{~A}$, which is enriched around the stop codon and in the $3^{\prime} \mathrm{UTR}$, m1A preferentially associates with the start codon region in mammalian $m R N A^{6}$. The unique chemical properties of $m 1 A$, with both a positive charge and a 
methyl group, potentially allow each modified site to have a stronger effect than m6A in terms of RNA structure or protein-RNA interactions ${ }^{4,7}$. Similar to the modification of DNA and protein, m1A modification is a kind of dynamic reversible process in mammalian cells that is regulated by methyltransferases, demethylases, and binding proteins, also known as "writers", "erasers" and "readers" 8 . The formation process of m1A methylation is catalyzed by methyltransferases consisting of TRMT10C, TRMT61B, TRMT6, and TRMT61 $A^{9,10}$, while the removal process is mediated by demethylases, including ALKBH1 and ALKBH $3^{11}$. In addition, a group of specific RNA-binding proteins composed of YTHDF1/2/3 and YTHDC1 can recognize the m1A motif, thus affecting m1A functions ${ }^{12}$. An in-depth understanding of these regulators would help reveal the role and mechanism of m1 A modification in posttranscriptional regulation.

In this study, using MM data from the Gene Expression Omnibus (GEO) database, we attempted to identify special m1A-related regulatory genes related to the progression of MM.

\section{Results}

\subsection{Landscape of genetic variations of $\mathrm{m} 1 \mathrm{~A}$ regulators in $\mathrm{MM}$}

We first summarized the differential expression of m1A regulators between normal plasma (NP, $n=9)$ and MM $(n=174)$ bone marrow plasma cells. Compared with NP, the expression of the eraser gene ALKBH1 was significantly downregulated in MM patients (Fig. 1A). The expression of the writer genes TRMT61A and TRMT61B and the reader genes YTHDF1, YTHDF2 and YTHDF3 were upregulated in MM patients.

Another GEO dataset $(n=559)$ with the most comprehensive clinical annotation was used to further investigate the prognostic values of $\mathrm{m} 1 \mathrm{~A}$ regulators. A univariate Cox regression model revealed the prognostic values of $10 \mathrm{~m} 1 \mathrm{~A}$ regulators in patients with MM. The Kaplan-Meier survival curve revealed seven regulators that were significantly correlated with survival. Among them, the high expression of six regulators (YTHDF1/2/3, TRMT6, TRMT10C, and TRMT61B), especially YTHDF2 and trmt61B, was associated with shorter overall survival (OS) (Fig. 1B-G). High expression of YTHDC1 was associated with longer OS (overall survival). The comprehensive landscape of $\mathrm{m} 1 \mathrm{~A}$ regulator interactions, regulator connections, and their prognostic significance in MM patients was depicted with the m1 A regulator network (Fig 1H). From the network diagram, we found that ALKBH1 was significantly negatively correlated with YTHDF2 and TRMT61B.

The above analyses indicated that the expression imbalance of m1A regulators, especially the high expression of three readers (YTHDF2, TRMT61B, and ALKBH1), may play a crucial role in MM occurrence and progression.

\subsection{M1A methylation modification patterns mediated by 10 regulators}


Three fully distinct modification patterns were eventually identified using unsupervised clustering, including 248 patients in pattern A and 190 patients in pattern B and 121 patients in pattern C. We termed these patterns m1Aclusters $A, B$, and $C$. Prognostic analysis of the three main m1A modification subtypes revealed a particular survival disadvantage in the m1Acluster B modification pattern (Fig. 2A). Clusters $A$ and $C$ were better than cluster $B$ in terms of survival and showed similar outcomes. There was a significant distinction in the $\mathrm{m} 1 \mathrm{~A}$ transcriptional profile between $\mathrm{m} 1 \mathrm{Aclusters} \mathrm{A}$ and $\mathrm{C}$ and $\mathrm{m} 1 \mathrm{Acluster} \mathrm{B}$ (Fig. 2B). As shown in Fig. 2C, cluster $B$ was characterized by the increased expression of five regulators (ALKBH3, YTHDF2, TRMT10C, TRMT6, and TRMT61B) and presented variable decreases in other m1 A regulators (Fig. $2 \mathrm{C}$ ). In contrast, $\mathrm{m} 1$ Acluster $\mathrm{C}$, with better survival, exhibited significant decreases in the expression of the same regulators. This probably means that these regulators, especially ALKBH3, YTHDF2, and TMRT61B, imply a poor prognosis.

To explore the biological behaviors related to these distinct m1 A modification patterns, we performed GSVA enrichment analysis. M1Aclusters $A$ and $C$ were markedly enriched in base excision repair and homologous recombination (Fig. 2D, E). M1Acluster B presented enrichment pathways associated with cell proliferation-related pathways, including nucleotide excision repair, DNA replication, aminoacyl tRNA biosynthesis, RNA polymerase, purine metabolism, and pyrimidine metabolism (Fig. 2D), suggesting that m1A modification may mediate the proliferation of MM cells.

\subsection{Generation of m1A gene signatures and functional annotation}

To further investigate the potential biological behavior of each $\mathrm{m} 1 \mathrm{~A}$ modification pattern, we identified 865 m1 A phenotype-related DEGs using the limma package (Fig. 3A). The Metascape platform was used to perform GO enrichment analysis for the DEGs. Surprisingly, these genes showed enrichment of biological processes remarkably related to the regulation of RNA biogenesis and metabolic processes (Fig. 3B), which confirmed again that $\mathrm{m} 1 \mathrm{~A}$ regulators played a nonnegligible role in RNA modification.

To further validate this regulatory mechanism, we then performed unsupervised clustering analyses based on the 865 obtained m1A phenotype-related genes to classify the patients into different genomic subtypes. Consistent with the clustering grouping of m1A modification patterns, the unsupervised clustering algorithm also revealed three distinct $\mathrm{m} 1 \mathrm{~A}$ modification genomic phenotypes, and we named these three clusters m1 A gene clusters A, B, and C (Fig. 3C). An intermediate prognosis was observed in gene cluster $A$, which included 273 patients. One hundred and twelve patients with MM were included in gene cluster $C$, which was proven to be related to a better prognosis. Patients in gene cluster $B(174$ patients) experienced poorer prognosis (Fig. 3D), with high expression of TRMT6, TRMT10C, TRMT61B, ALKBH3, and YTHDF1/2/3 and low expression of ALKBH1 and YTHDC1, especially TRMT61B, YTHDF2, ALKBH3 and ALKBH1 (Fig. 3E), as mentioned above. In the three m1A gene clusters, prominent differences in the expression of $\mathrm{m} 1 \mathrm{~A}$ regulators were observed, which was in accordance with the expected results of m1A methylation modification patterns. 
To further identify the prognostic value of the m1Ascore, the patients were divided into low- and highm1 Ascore groups with a cutoff value of -2.3971 using the survminer package. The Kruskal-Wallis test revealed that patients with a low $\mathrm{m} 1$ Ascore had inferior survival (Fig. 4A). Then, we specifically examined the ability of the $\mathrm{m} 1$ Ascore to serve as a prognostic biomarker for patients with $\mathrm{MM}$. Gene cluster $\mathrm{B}$ showed the lowest median score, and m1Acluster B also showed the lowest median score (Fig. 4B-C). Compared with patients with low m1Ascores, patients with high m1Ascores had a prominent survival probability (74\% vs. $63 \%$ ), which again proved that low m1Ascores could be closely linked to poor prognosis (Fig. 4D). In addition, patients with high m1Ascores showed obviously higher expression levels of PD-L1, which indicated a potential response to anti-PD-L1 immunotherapy (Fig. 4E).

\section{Discussion}

M1A modification is widely distributed in RNA and has been proven to play an important role in ensuring the correct folding of tRNAs and promoting the formation of rRNA subunits ${ }^{17,18}$. In mRNA, m1A was found to be highly enriched in the $5^{\prime}$ UTR and tended to be located in highly structured regions ${ }^{6}$. In addition, some studies identified an m1A modification site in MALAT $1{ }^{9}$, a IncRNA that could be employed in cancer diagnosis and prognosis ${ }^{19}$. Increasing evidence suggests that changes in m1A-related regulatory genes are closely associated with a variety of diseases, including gastrointestinal cancer and glioblastoma ${ }^{20,21}$. However, the role of $\mathrm{m} 1 \mathrm{~A}$ methylation in the occurrence and prognosis of MM remains unclear.

By exploring the GEO database, we analyzed a total of 183 samples $(\mathrm{NP}=9, \mathrm{MM}=174)$ to understand alterations in $\mathrm{m} 1 \mathrm{~A}$-related regulator genes in the $\mathrm{MM}$ samples. The expression of five $\mathrm{m} 1 \mathrm{~A}$ regulatory genes was upregulated in MM patients, including the writer TRMT61A/B and the readers YTHDF1/2/3. The expression of the eraser ALKBH1 was significantly downregulated. Then, the Kaplan-Meier curve showed that the expression of m1A-related regulatory genes could be used to assess MM patient risk, especially the high expression of TRMT6, TRMT61B, and YTHDF1/2/3 associated with shorter overall survival and distinct situations of YTHDC1. This result suggested that the expression of m1A-related regulatory genes could be a useful prognostic marker for $\mathrm{MM}$. Cluster analysis revealed three distinct $\mathrm{m} 1 \mathrm{~A}$ modification patterns based on the expression of $10 \mathrm{~m} 1 \mathrm{~A}$ regulators. M1Acluster $\mathrm{B}$ was characterized by the increased expression of ALKBH3, YTHDF2, TRMT10C, TRMT6, and TRMT61B and presented apparent decreases in ALKBH1 and YTHDC1, with the worst outcome. M1Acluster $\mathrm{C}$ with better survival exhibited significant contrary changes in the expression of these regulators, which means that these regulators (especially ALKBH1/3, TRMT61B, and YTHDF2) have the potential to serve as important biomarkers for MM. In our study, the GSVA results revealed the biological functions of m1A-related regulatory genes involved in $\mathrm{MM}$, which were related to the proliferation of cells, including nucleotide excision repair, DNA replication, aminoacyl tRNA biosynthesis, RNA polymerase, purine metabolism, and pyrimidine metabolism, which provide clues to their contribution to MM pathogenesis. 
The next unsupervised cluster analysis showed three distinct m1A modification genomic phenotypes consistent with the m1A modification patterns. In accordance with the expected results of the m1A modification patterns, the expression of m1A regulators (high expression of TRMT6, TRMT61B, YTHDF2, and ALKBH3 and low expression of ALKBH1 and YTHDC1) was also observed in the m1A gene clusters. Patients in $\mathrm{m} 1 \mathrm{~A}$ gene cluster $\mathrm{B}$ experienced the worst prognosis, while those in gene cluster $\mathrm{C}$ showed the best prognosis, which confirmed that the ALKBH3, TRMT61B, YTHDF2, and ALKBH1 genes had good risk prediction value for MM survival, and elevated expression of the previous three regulators was associated with poor prognosis.

In addition to nuclear-encoded transcripts, M1A modification also exists in mitochondrial-coded mRNAs (mt-mRNAs) ${ }^{22}$. TRMT61B is a mitochondria-located methyltransferase that can modify not only mt-tRNA and mt-rRNA but also mt-mRNA ${ }^{23}$. YTHDF2 is overexpressed in a broad spectrum of human AMLs and contributes to the integrity of leukemia stem cell function. Therefore, YTHDF2 is considered a unique target for the treatment of $\mathrm{AML}^{26}$.

From the 10 regulators network diagram, we found a strong positive correlation between YTHDF2 and TRMT61B, and they were significantly negatively correlated with ALKBH1. A previous study revealed the significant effects of ALKBH1 on subsequent translational initiation, which results in attenuated translation initiation and reduces the role of target tRNAs in protein synthesis ${ }^{27}$. The demethylases $A L K B H 1$ and $A L K B H 3$ have been characterized as $m 1 A$ erasers ${ }^{28}$. However, we found that the expression of ALKBH1 and ALKBH3 had opposite effects on the prognosis of MM patients. Human ALKBH3 belongs to the AlkB family that utilizes nonheme iron (II) to catalyze biological oxidation and could participate in the demethylation of single-stranded RNA both in vitro and in vivo ${ }^{27,29,30}$. Previous research shows that hypermethylation of the ALKBH3 promoter $\mathrm{CpG}$ island is common in hematological malignancies, and ALKBH3 hypermethylation is associated with shorter OS in Hodgkin lymphoma ${ }^{31}$. This result is consistent with the upregulation of ALKBH3 in patients with poor prognosis found in our study.

The Kruskal-Wallis test revealed significant and similar differences in m1A scores in the m1A clusters and m1 A gene clusters. Cluster B presented the lowest median score compared to the other clusters, and cluster $\mathrm{C}$ had the highest median score. Combining the above analysis with the $\mathrm{m} 1$ Ascore survival curve, we found that patients with high m1Ascores had a prominent survival benefit. To test whether the m1 Ascore could serve as an independent prognostic biomarker for MM, we next confirmed that patients with high $\mathrm{m} 1$ Ascores had a higher survival rate. We also identified the predictive value of the $\mathrm{m} 1$ Ascore for anti-PD-L1 immunotherapy, in which a high m1Ascore indicated higher expression of PD-L1.

\section{Conclusions}

Our study identified alterations in m1A-related regulatory genes in MM for the first time and found a clear relationship with clinicopathological features and prognosis. ALKBH3, TRMT61B, YTHDF2, and ALKBH1 effectively predicted MM patient survival and contributed to important biological processes. Similarly, the constructed m1Ascore was found to be a prognostic biomarker for patients with MM and could predict 
the patients' clinical response to PD-L1 immunotherapy. To further clarify the molecular mechanism of m1 A mRNA modification in MM development, future studies in vivo and in vitro are needed to confirm our findings and molecular understanding.

\section{Methods}

\subsection{MM dataset source and preprocessing}

Public gene expression data and full clinical annotation were searched in the GEO database. In total, 3 eligible MM cohorts (GSE13591, GSE47552, and GSE24080) were gathered in this study for further analysis, and the normalized matrix files were directly downloaded from platforms. The data were analyzed with R (version 4.1.2) and R Bioconductor packages.

\subsection{Analysis of m1A-related regulatory gene expression}

We first analyzed the mRNA expression of m1A regulators in normal plasma and MM bone marrow plasma cells based on samples from GSE13591 and GSE47552. Then, another dataset (GSE24080) with prolific clinical information was used to elucidate alterations of m1A-related regulatory genes in MM samples. The data were analyzed with the limma R package.

\subsection{Unsupervised clustering for $10 \mathrm{~m} 1 \mathrm{~A}$ regulators}

A total of 10 regulators were extracted from GSE24080 to identify different m1A modification patterns mediated by $\mathrm{m} 1 \mathrm{~A}$ regulators. These $10 \mathrm{~m} 1 \mathrm{~A}$ regulators included 4 writers (TRMT6, TRMT10C, TRMT61A, and TRMT61B), 2 erasers (ALKBH1 and ALKBH3) and 4 readers (YTHDC1, YTHDF1, YTHDF2, and YTHDF3). Unsupervised clustering analysis was applied to identify distinct m1A modification patterns based on the expression of $10 \mathrm{~m} 1 \mathrm{~A}$ regulators and classify patients for further analysis. The number of clusters and their stability were determined by the consensus clustering algorithm ${ }^{13}$. The ConsensusClusterPlus package was used to perform the above steps.

\subsection{Gene set variation analysis (GSVA) and functional annotation}

To investigate the difference in biological processes between $\mathrm{m} 1 \mathrm{~A}$ modification patterns, we performed GSVA enrichment analysis using the GSVA R package. GSVA is a nonparametric and unsupervised method and can easily adapt to the analysis of RNA-seq data ${ }^{14}$. The gene sets of "c2.cp.kegg.v6.2.symbols" were downloaded from the MSigDB database for GSVA. Adjusted P values less than 0.05 were considered statistically significant. The clusterProfiler $\mathrm{R}$ package was used to perform functional annotation for m1A-related genes, with the cutoff values of | log fold change (FC) $\mid>0.1$ and adjusted $P$ value $<0.05$.

\subsection{Identification of differentially expressed genes (DEGs) between distinct m1A phenotypes}


To further analyze m1A-related regulators, we identified DEGs based on different modification patterns of $\mathrm{m} 1 \mathrm{~A}$. The limma R package was applied to identify DEGs, and the significance criteria were set as adjusted $P$ value $<0.001$. Gene Ontology (GO) enrichment analysis of DEGs was performed by the Metascape platform.

\subsection{Generation of an $\mathrm{m} 1 \mathrm{~A}$ gene signature}

To preferably quantify the m1 A modification pattern, we constructed the $\mathrm{m} 1 \mathrm{~A}$ gene signature to calculate a series of scores to assess individual MM patients, and it was termed the m1 Ascore. The steps for establishing the m1A gene signature were as follows.

We first extracted overlapping genes from DEGs identified from different m1 Aclusters and then performed unsupervised clustering analysis on the overlapping genes. The consensus clustering algorithm was used to determine the number and stability of gene clustering, and the patients were divided into several groups for further analysis. Next, we used univariate regression analysis to analyze the prognosis of each overlapping gene and extracted the genes with significant prognoses for further analysis.

Principal components 1 and 2 were selected as signature scores, and $\mathrm{m} 1 \mathrm{~A}$-related gene signatures were constructed through principal component analysis (PCA).

We then defined the m1Ascore using a method similar to the gene expression grade index $(\mathrm{GGI})^{15,16}$.

m1Ascore $=\sum\left(P C 1_{i}+P C 2_{i}\right)$

where $\mathrm{i}$ is the expression of $\mathrm{m} 1 \mathrm{~A}$ phenotype-related genes.

\subsection{Statistical analysis}

The survival curves for the prognostic analysis were generated via the Kaplan-Meier method, and logrank tests were used to identify the significance of differences. We adopted a univariate Cox regression model to calculate the hazard ratios (HRs) for $\mathrm{m} 1 \mathrm{~A}$ regulators and $\mathrm{m} 1 \mathrm{~A}$ phenotype-related genes. The independent prognostic factors were ascertained through a multivariable Cox regression model. Patients with detailed clinical data were eligible for inclusion in the final multivariate prognostic analysis. All statistical $P$ values were two-sided, with $P<0.05$ considered statistically significant. All data processing was performed in R 4.1.2 software.

\section{Abbreviations}

$\mathrm{M} 1 \mathrm{~A}=\mathrm{N1}$-methyladenosine; $\mathrm{MM}=$ Multiple myeloma; $\mathrm{NP}=$ normal plasma; $\mathrm{m5C}$ = 5-methylcytosine; m6A = N6-methyladenosine; GEO = Gene Expression Omnibus; OS = overall survival; GSVA = Gene set variation analysis; $\mathrm{FC}=$ fold change; $\mathrm{DEGs}=$ differentially expressed genes; $\mathrm{GO}=$ Gene Ontology; $\mathrm{PCA}=$ principal component analysis; $\mathrm{GGI}$ = gene expression grade index; HRs = hazard ratios .

\section{Declarations}




\section{Ethical Approval and Consent to participate}

Not applicable.

\section{Consent for publication}

Not applicable.

\section{Availability of data and materials}

The datasets generated during the current study are available in the Gene Expression Omnibus (GEO) database.

\section{Competing interests}

The authors report no conflicts of interest related to this study.

\section{Funding}

The study was supported by the National Natural Science Foundation of China (No.

82074348).

\section{Authors' contributions}

Fu Jiaqi and Cui Xing wrote the main manuscript text. Fu Jiaqi and Yu Manya prepared figures 1-4. Han Xingjun, Gao Wei and Cui Jinwei did the preparatory work. All authors reviewed the manuscript.

\section{Acknowledgements}

Not applicable.

\section{References}

1. Zhan F, Huang Y, Colla S, et al. The molecular classification of multiple myeloma. Blood. 2006;108(6):2020-8.

2. Röllig C, Knop S, Bornhäuser M. Multiple myeloma. The Lancet. 2015;385 (9983):2197-2208.

3. Pawlyn C, Davies FE. Toward personalized treatment in multiple myeloma based on molecular characteristics. Blood. 2019;133(7):660-675.

4. Zhao BS, Roundtree IA, He C. Post-transcriptional gene regulation by mRNA modifications. Nat Rev Mol Cell Biol. 2017;18(1):31-42. 
5. Li X, Xiong X, Wang K, et al. Transcriptome-wide mapping reveals reversible and dynamic N(1)methyladenosine methylome. Nat Chem Biol. 2016;12(5):311-6.

6. Dominissini D, Nachtergaele S, Moshitch-Moshkovitz S, et al. The dynamic N(1)-methyladenosine methylome in eukaryotic messenger RNA. Nature. 2016;530(7591):441-6.

7. Meyer KD, Saletore $Y$, Zumbo P, et al. Comprehensive analysis of mRNA methylation reveals enrichment in 3' UTRs and near stop codons. Cell. 2012;149(7):1635-46.

8. Yang Y, Hsu PJ, Chen YS, et al. Dynamic transcriptomic m6A decoration: writers, erasers, readers and functions in RNA metabolism. Cell Res. 2018;28(6):616-624.

9. Safra M, Sas-Chen A, Nir R, et al. The m1A landscape on cytosolic and mitochondrial mRNA at singlebase resolution. Nature. 2017;551(7679):251-255.

10. Chujo T, Suzuki T. Trmt61B is a methyltransferase responsible for 1-methyladenosine at position 58 of human mitochondrial tRNAs. RNA. 2012;18(12):2269-76.

11. Chen Z, Qi M, Shen B, et al. Transfer RNA demethylase ALKBH3 promotes cancer progression via induction of tRNA-derived small RNAs. Nucleic Acids Res. 2019;47(5):2533-2545.

12. Dai X, Wang T, Gonzalez G, et al. Identification of YTH Domain-Containing Proteins as the Readers for N1-Methyladenosine in RNA. Anal Chem. 2018;90(11):6380-6384.

13. Li J, Xie L, Xie Y, et al. Bregmannian consensus clustering for cancer subtypes analysis. Comput Methods Programs Biomed. 2020;189:105337.

14. Hänzelmann S, Castelo R, Guinney J. GSVA: gene set variation analysis for microarray and RNA-seq data. BMC Bioinformatics. 2013;14:7.

15. Sotiriou C, Wirapati P, Loi S, et al. Gene expression profiling in breast cancer: understanding the molecular basis of histologic grade to improve prognosis. J Natl Cancer Inst. 2006;98(4):262-72.

16. Zhang B, Wu Q, Li B, et al. m6A regulator-mediated methylation modification patterns and tumor microenvironment infiltration characterization in gastric cancer. Mol Cancer. 2020;19(1):53.

17. Waku T, Nakajima Y, Yokoyama W, et al. NML-mediated rRNA base methylation links ribosomal subunit formation to cell proliferation in a p53-dependent manner. J Cell Sci. 2016;129(12):2382-93.

18. Voigts-Hoffmann F, Hengesbach M, Kobitski AY, et al. A methyl group controls conformational equilibrium in human mitochondrial tRNA(Lys). J Am Chem Soc. 2007;129(44):13382-3.

19. Goyal B, Yadav SRM, Awasthee N, et al. Diagnostic, prognostic, and therapeutic significance of long non-coding RNA MALAT1 in cancer. Biochim Biophys Acta Rev Cancer. 2021;1875(2):188502. 
20. Dong Z, Cui H. The Emerging Roles of RNA Modifications in Glioblastoma. Cancers (Basel). 2020;12(3):736.

21. Zhao Y, Zhao Q, Kaboli PJ, et al. m1A Regulated Genes Modulate PI3K/AKT/mTOR and ErbB Pathways in Gastrointestinal Cancer. Transl Oncol. 2019;12(10):1323-1333.

22. Xiong X, Li X, Yi C. N1-methyladenosine methylome in messenger RNA and non-coding RNA. Curr Opin Chem Biol. 2018;45:179-186.

23. Li X, Xiong X, Zhang M, et al. Base-Resolution Mapping Reveals Distinct m1A Methylome in Nuclearand Mitochondrial-Encoded Transcripts. Mol Cell. 2017;68(5):993-1005.

24. Sekar S, McDonald J, Cuyugan L, et al. Alzheimer's disease is associated with altered expression of genes involved in immune response and mitochondrial processes in astrocytes. Neurobiol Aging. 2015;36(2):583-91.

25. Seo KW, Kleiner RE. YTHDF2 Recognition of N1-Methyladenosine (m1A)-Modified RNA Is Associated with Transcript Destabilization. ACS Chem Biol. 2020;15(1):132-139.

26. Paris J, Morgan M, Campos J, et al. Targeting the RNA m6A Reader YTHDF2 Selectively Compromises Cancer Stem Cells in Acute Myeloid Leukemia. Cell Stem Cell. 2019;25(1):137-148.

27. Liu F, Clark W, Luo G, et al. ALKBH1-Mediated tRNA Demethylation Regulates Translation. Cell. 2016;167(3):816-828.

28. Aas PA, Otterlei $\mathrm{M}$, Falnes $\mathrm{PO}$,et al. Human and bacterial oxidative demethylases repair alkylation damage in both RNA and DNA. Nature. 2003;421(6925):859-63.

29. Ougland R, Zhang CM, Liiv A, et al. AlkB restores the biological function of mRNA and tRNA inactivated by chemical methylation. Mol Cell. 2004;16(1):107-16.

30. Trewick SC, Henshaw TF, Hausinger RP, et al. Oxidative demethylation by Escherichia coli AlkB directly reverts DNA base damage. Nature. 2002;419(6903):174-8.

31. Esteve-Puig R, Climent F, Piñeyro D, et al. Epigenetic loss of m1A RNA demethylase ALKBH3 in Hodgkin lymphoma targets collagen, conferring poor clinical outcome. Blood. 2021;137(7):994-999.

\section{Figures}


A

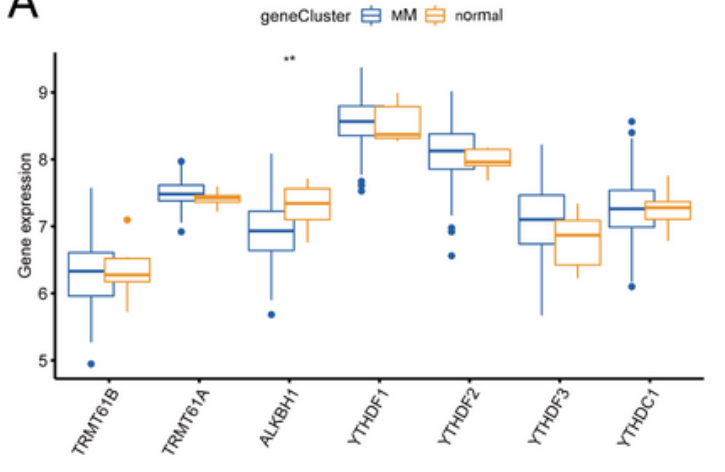

C

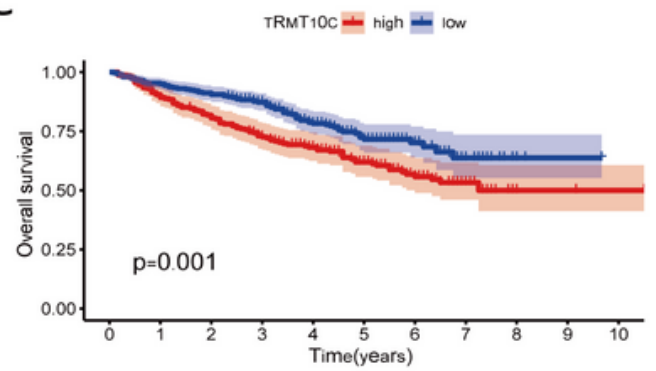

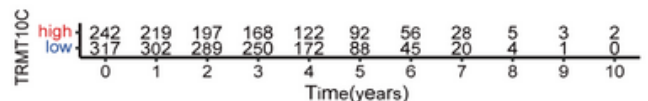

$\mathrm{E}$

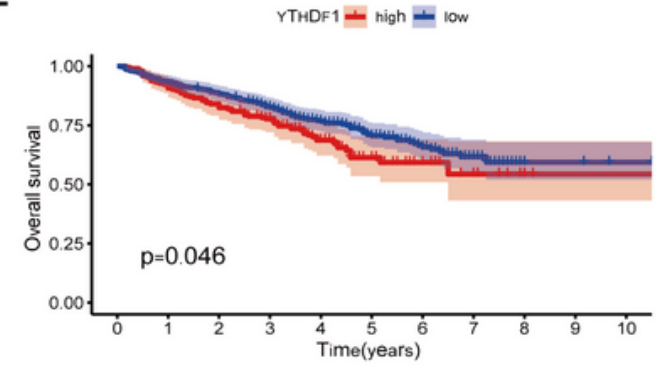

B

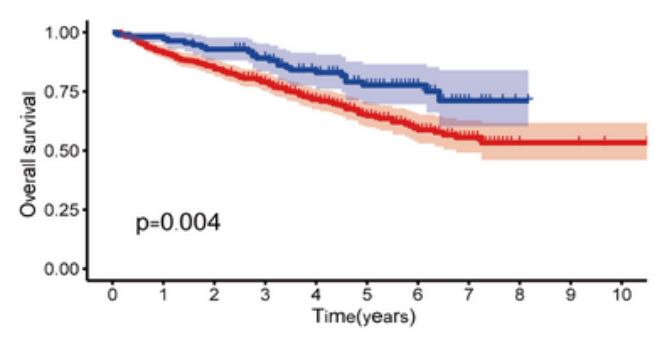

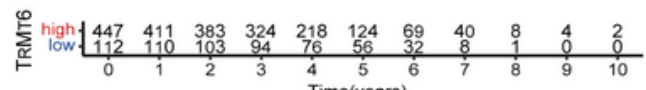

D
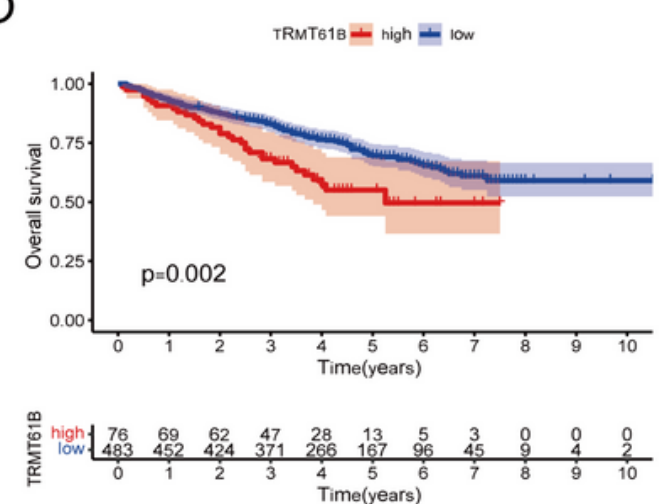

$\mathrm{F}$

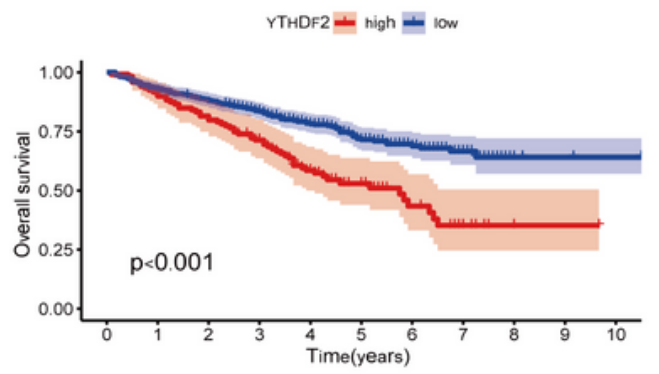

G

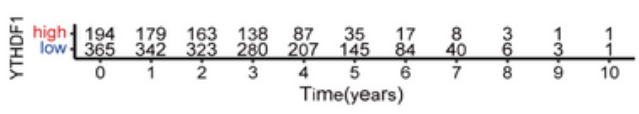

$\mathrm{H}$

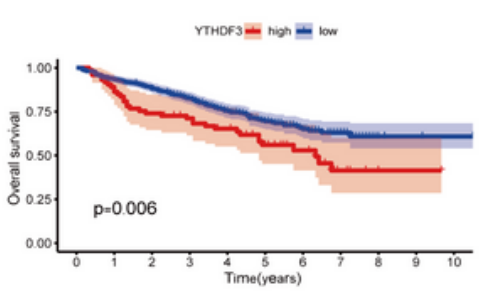

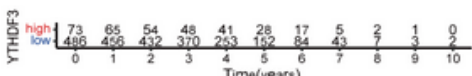

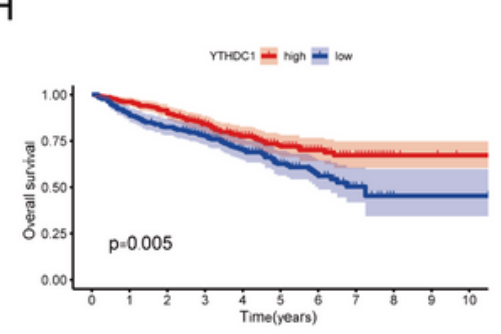

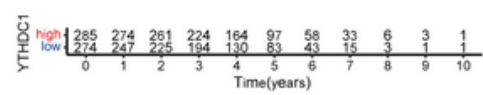

I

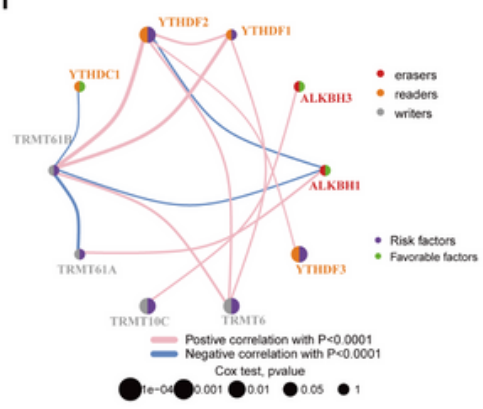

\section{Figure 1}

\section{Landscape of genetic variations of $\mathrm{m} 1 \mathrm{~A}$ regulators in multiple myeloma.}

(A) Alterations in m1A-related regulatory genes in MM patients. The upper and lower ends of the boxes represent an interquartile range of values. The lines in the boxes represent the median value, and black dots show outliers. The asterisks represent significant $P$ values $(P<0.1, * * P<0.01)$. (B-G) High expression 
of (B) TRMT6, (C) TRMT10C, (D) TRMT61B, (E) YTHDF1, (F) YTHDF2 and (G) YTHDF3 is associated with poor prognosis in HCC patients. $(\mathrm{H})$ Low expression of YTHDC1 is associated with a better prognosis. (I) The interaction between $\mathrm{m} 1 \mathrm{~A}$ regulators in $\mathrm{MM}$. The circle size represents the effect of each regulator on the prognosis, and the range of values calculated by the log-rank test was $P<0.001, P<0.01, P<0.05$ and $P<0.1$. Purple dots in the circle indicate risk factors for prognosis; green dots in the circle indicate protective factors for prognosis. The lines linking regulators showed their interactions, and the thickness shows the correlation strength between regulators. Negative correlations are marked with blue, and positive correlations are marked with red. The regulator erasers, readers and writers are marked with red, orange and gray, respectively.

\section{Figure 2}

\section{M1A methylation modification patterns mediated by 10 regulators.}

(A) Survival analyses for the three m1A modification patterns based on 599 patients with gastric cancer from GSE24080. Kaplan-Meier curves with a log-rank $P$ value of 0.002 showed a significant survival difference among the three $\mathrm{m} 1 \mathrm{~A}$ modification patterns. m1Acluster $\mathrm{B}$ showed worse overall survival than the other two m1Aclusters. (B) Principal component analysis of the transcriptome profiles of the three m1A modification patterns, showing a remarkable difference in the transcriptome between different modification patterns. (C) Unsupervised clustering of $10 \mathrm{~m} 1 \mathrm{~A}$ regulators. The $\mathrm{m} 1$ Acluster, ISS staging system, sex, immunoglobulin type and age were used as patient annotations. Red represents high expression of regulators, and blue represents low expression. (D-E) GSVA enrichment analysis showed the activation states of biological pathways in distinct m1A modification patterns. A heatmap was used to visualize these biological processes; red represents activated pathways, and blue represents inhibited pathways. The MM cohort was used as sample annotations. (D) m1Acluster A vs. m1Acluster B; (E) m1 Acluster B vs. m1Acluster C. 
A
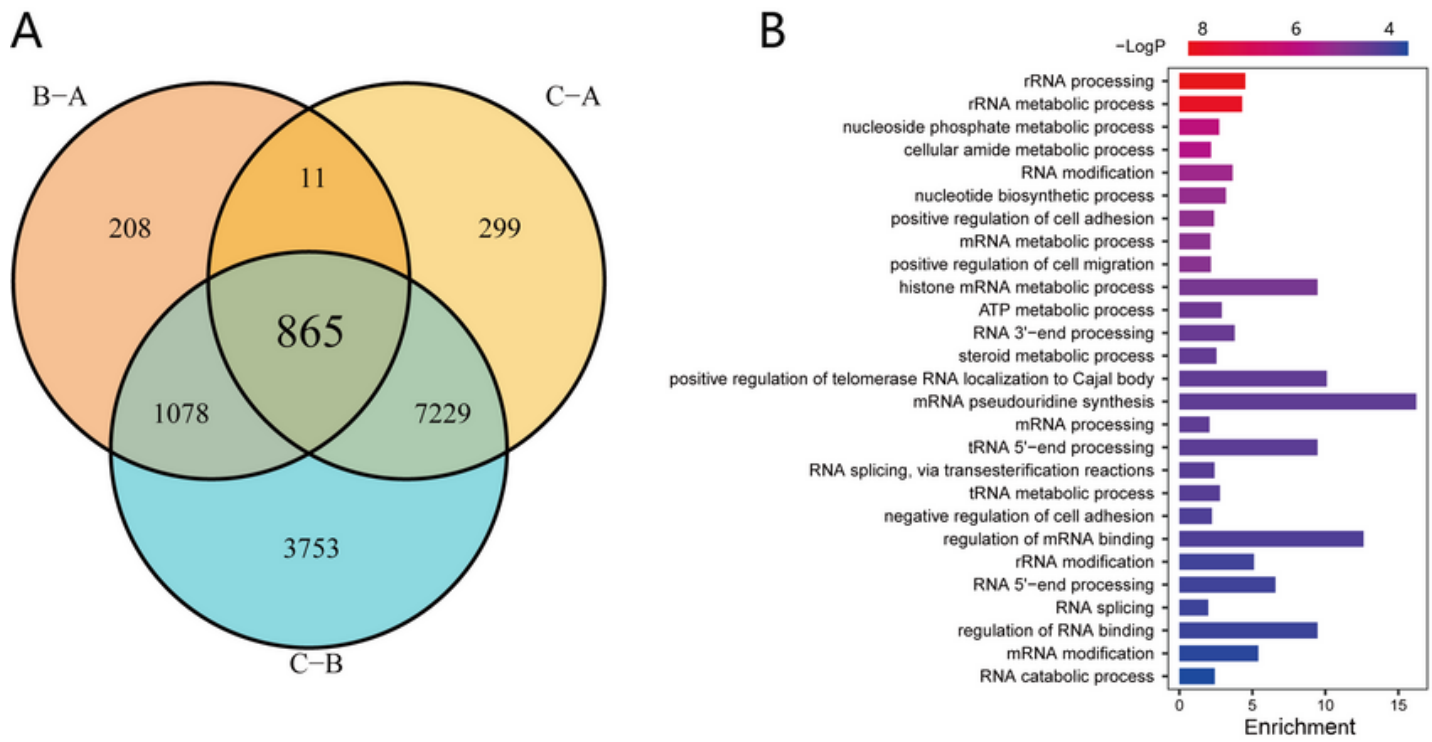

C

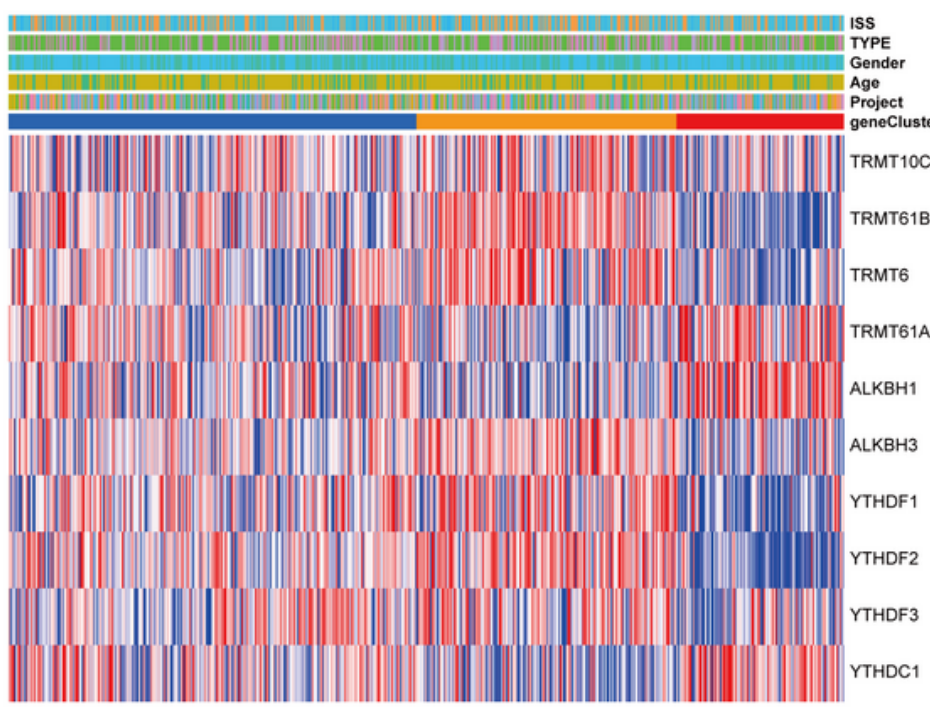

D
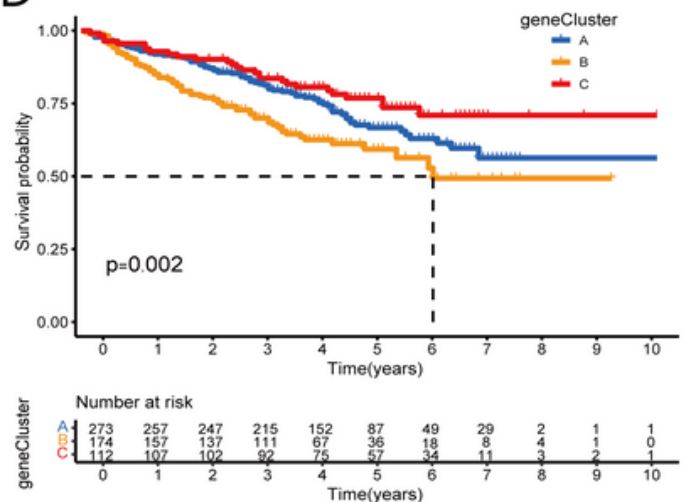

$\mathrm{E}$

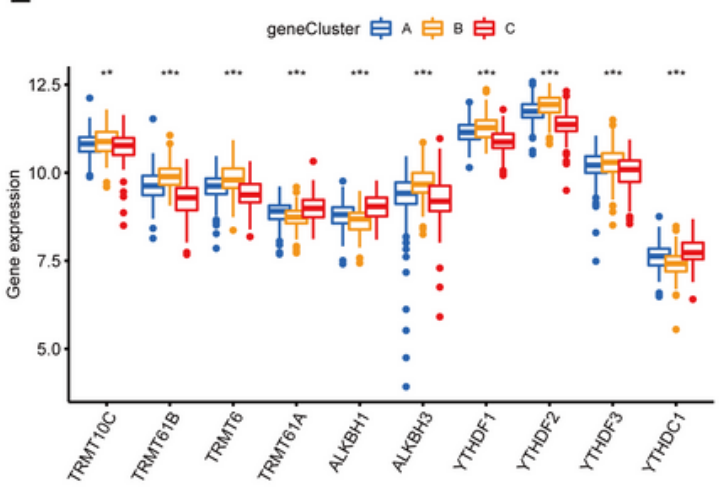

\section{Figure 3}

\section{Generation of m1A gene signatures and functional annotation.}

(A) Identification of differentially expressed genes (DEGs) between distinct m1A phenotypes. (B) Functional annotation for m1A-related genes using GO enrichment analysis. The color depth of the bar plots represents the number of enriched genes. (C) Unsupervised clustering of overlapping m1A 
phenotype-related genes to classify patients into different genomic subtypes, termed m1A gene clusters A-C. m1Acluster, ISS staging system, sex, immunoglobulin type and age were used as patient annotations. (D) Kaplan-Meier curves indicated that m1A modification genomic phenotypes were markedly related to the overall survival of 559 patients, of which 273 patients were in gene cluster $A, 174$ patients were in gene cluster $B$, and 112 patients were in gene cluster $C(P<0.0001$, log-rank test). (E) The expression of $10 \mathrm{~m} 1 \mathrm{~A}$ regulators in three gene clusters. The upper and lower ends of the boxes represent the interquartile range of values. The lines in the boxes represent the median value, and black dots show outliers. The asterisks represent significant $P$ values $\left({ }^{*} P<0.05 ;{ }^{* *} P<0.01 ; * * * P<0.001\right)$.
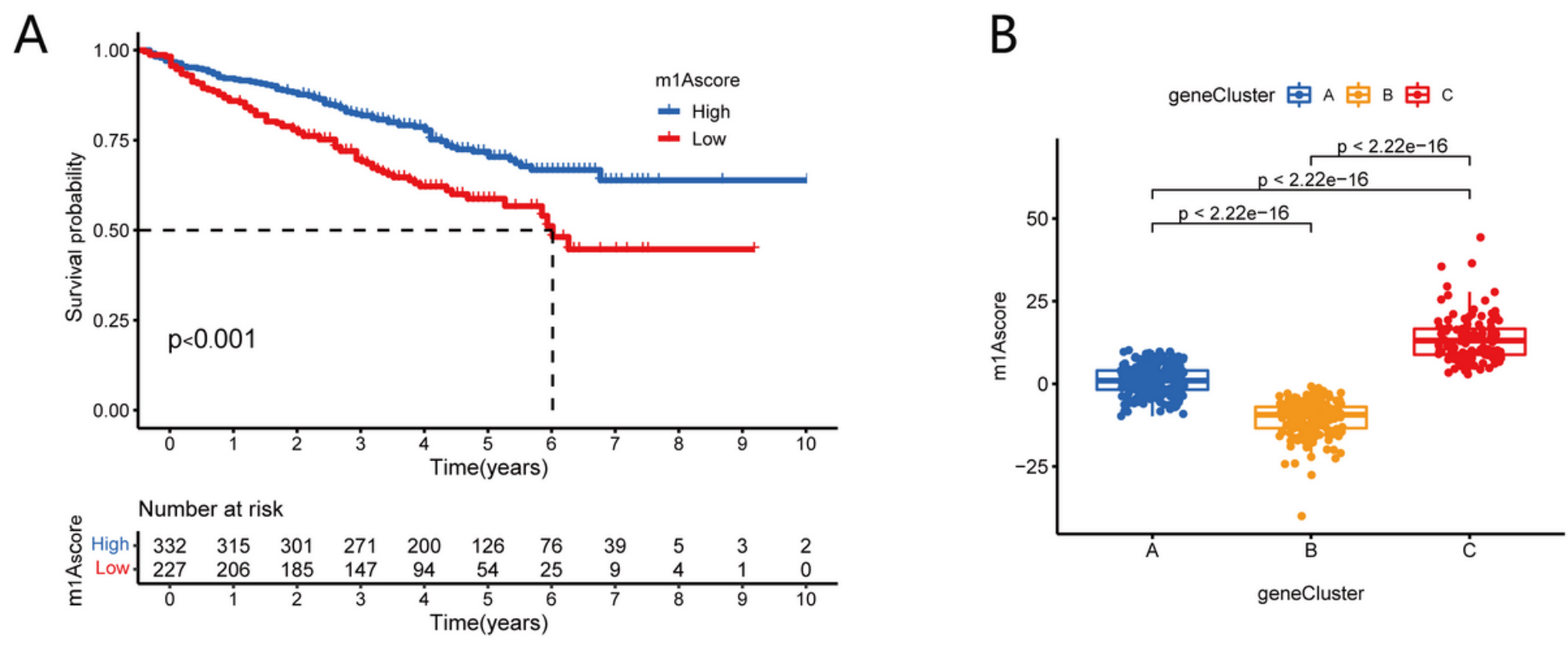

C
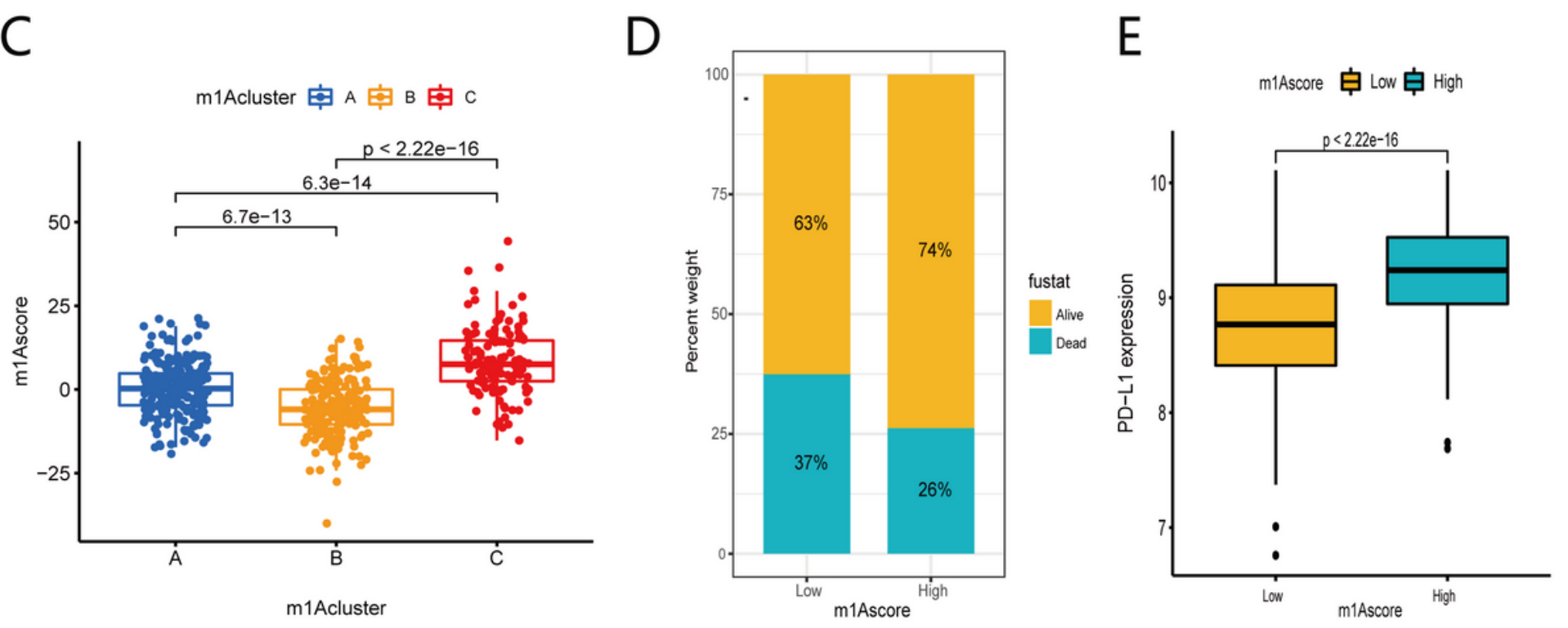

Figure 4

Prognostic values of m1Ascore in multiple myeloma.

(A) Survival analyses for the low-m1Ascore (227 patients) and high-m1Ascore (332 patients) patient groups $(P<0.001)$. (B) Differences in the m1Ascore among the three gene clusters. (C) Differences in the 
m1 Ascore among the three m1A clusters. (D) The proportion of patients who survived in the low- or high$\mathrm{m} 1$ Ascore groups. Alive/dead $63 \% / 37 \%$ in the low-m1Ascore groups and $74 \% / 26 \%$ in the high-m1 Ascore groups. (E) Differences in PD-L1 expression between the low- and high-m1Ascore groups $(P<2.22 \mathrm{e}-16$, Wilcoxon test). 